\title{
Efecto de la temperatura en el desarrollo embrionario y larval del mejillón, Mytilus galloprovincialis (Lamarck, 1819)
}

\author{
Temperature effect in the embryonic and larval development of the mussel, \\ Mytilus galloprovincialis (Lamarck, 1819)
}

\section{Maryori Ruiz $^{1,2}$, Eduardo Tarifeño ${ }^{2,3}$, Alejandra Llanos-Rivera ${ }^{3}$, Christa Padget $^{3}$ y Bernardita Campos ${ }^{4}$}

\author{
${ }^{1}$ Programa de Magíster en Ciencias, Departamento Zoología, Facultad de Ciencias Naturales y Oceanográficas, \\ Universidad de Concepción. Casilla 160-C, Concepción, Chile \\ ${ }^{2}$ Departamento de Zoología, Facultad de Ciencias Naturales y Oceanográficas, \\ Universidad de Concepción, Casilla 160-C, Concepción, Chile \\ ${ }^{3}$ Grupo ProMytilus. Proyecto FONDEF D03-1095, Facultad de Ciencias Naturales y Oceanográficas, \\ Universidad de Concepción, Chile \\ ${ }^{4}$ Facultad de Ciencias del Mar y de Recursos Naturales, Universidad de Valparaíso, Valparaíso, Chile \\ Casilla 5080, Reñaca, Viña del Mar, Chile \\ etarifen@udec.cl
}

\begin{abstract}
The Mediterranean mussel (Mytilus galloprovincialis Lamarck, 1819) has been recently registered for the Chilean coast, from Concepcion $\left(36^{\circ} \mathrm{S}\right)$ to the Magellan Strait $\left(54^{\circ} \mathrm{S}\right)$. To determine the feasibility of the massive culture of M. galloprovincialis in Chile, a study of the temperature effects on the early development was carried out. Embryos and larvae were raised at 12,16 and $20^{\circ} \mathrm{C}$ in laboratory facilities at Coliumo Bay $\left(36^{\circ} \mathrm{S}\right)$, Chile, from where adults mussels were collected. Embryonic development followed the common sequence exhibited by other bivalves. Trocophores turned into veligers at $45 \mathrm{~h}$ post fertilization, followed by D-larvae $(99 \pm$ $6 \mu \mathrm{m}$ length, initial size), with a large ciliated velum, a welldeveloped stomach and concentric growth lines in the shell; 15 days post-fertilization, umbonate larvae developed eye-spots and adductors muscles; pediveliger larvae $(265 \pm 2 \mu \mathrm{m}$ initial length size) developed a pedal organ and gill filaments. Both embryonic and larval stages were accelerated at higher temperatures. The larval growth rates at $20^{\circ} \mathrm{C}\left(9.1 \mu \mathrm{m}^{*} \mathrm{~d}^{-1}\right)$ were significantly larger than at 12 and $16^{\circ} \mathrm{C}\left(6.7 \mu \mathrm{m}^{*} \mathrm{~d}^{-1}\right.$ and 7.4 $\mu \mathrm{m}^{*} \mathrm{~d}^{-1}$, respectively). In comparison with Mytilus chilensis, M. galloprovincialis showed better growth rates at the same temperatures. This fact suggests that in Chile $M$. galloprovincialis seed can be produced under controlled conditions for its massive cultivation.
\end{abstract}

Key words: Mytilidae, mussel, embryo, larvae, temperature, growth rate
Resumen.- El mejillón del Mediterráneo (Mytilus galloprovincialis Lamarck, 1819) ha sido registrado recientemente en las costas chilenas, desde Concepción $\left(36^{\circ} \mathrm{S}\right)$ hasta el Estrecho de Magallanes (54 $\mathrm{S})$. En el estudio de factibilidad de su cultivo masivo en Chile (Proyecto FONDEF D05I-10258) se evaluó el efecto de la temperatura sobre su desarrollo temprano para modular empíricamente las tasas de crecimiento larval. Los embriones y larvas se cultivaron a 12 , 16 y $20^{\circ} \mathrm{C}$, en una unidad productora de semillas marinas en la bahía de Coliumo ( $\left.36^{\circ} \mathrm{S}\right)$, Chile, desde donde se extrajeron los reproductores. El desarrollo embrionario siguió la secuencia conocida para otros bivalvos. Las trocóforas se transformaron en velígeras, a las $45 \mathrm{~h}$ post fertilización, seguidas por las larvas $\mathrm{D}$ (longitud inicial $99 \pm 6 \mu \mathrm{m}$ ), con velo ciliado, estómago bien definido y líneas de crecimiento concéntricas en la concha; las larvas umbonadas, a partir del día 15 post fertilización, desarrollaron manchas oculares y músculos aductores; las pedivelígeras (talla inicial $265 \pm 2 \mu \mathrm{m}$ ) desarrollaron un órgano pedal y filamentos branquiales. Los estadios embrionario y larval fueron acelerados a mayores temperaturas. Las tasas de crecimiento larval a $20^{\circ} \mathrm{C}\left(9,1 \mu \mathrm{m}^{*} \mathrm{día}^{-1}\right)$ fueron significativamente mayores que a 12 y $16^{\circ} \mathrm{C}\left(6,7 \mu \mathrm{m}^{*} \mathrm{dí}^{-1} \mathrm{y}\right.$ 7,4 $\mu \mathrm{m}^{*} \mathrm{dí}^{-1}$, respectivamente). Comparado con Mytilus chilensis, M. galloprovincialis presentó mejores de tasas de crecimiento a iguales temperaturas. Esto sugiere que, en Chile, se puede producir semillas de M. galloprovincialis en condiciones controladas, para su cultivo masivo.

Palabras clave: Mytilidae, mejillón, embrión, larva, temperatura, tasa de crecimiento 


\section{Introducción}

La mitilicultura en Chile ha experimentado un acelerado crecimiento en el quinquenio 2000-2005. De las especies cultivadas (Mytilus edulis chilensis, Aulacomya atra y Choromytilus chorus) sólo M. edulis chilensis muestra una producción masiva, pasando desde 30 mil toneladas anuales en 2003 hasta unas 88 mil en 2005 (SERNAPESCA 2005), con planes de expansión hasta las 300 mil toneladas el 2010. Los motivos de este notable incremento han sido la constante demanda insatisfecha (11\%) por mejillones en los mercados mundiales, debido a la imposibilidad del crecimiento de la oferta española por limitación en números de bateas y cierre de polígonos por contaminación y floraciones nocivas (Blanco et al. 2002). El crecimiento reciente de la mitilicultura chilena se ha basado en: el aumento de las concesiones de acuicultura para operar centros de crecimiento y engorda, el aumento de la superficie productiva y del número de los sistemas de cultivo en actual explotación, y el aumento de la productividad de los sistemas desde un nivel artesanal hacia uno más industrializado (SUBPESCA 2005).

La reciente constatación de la presencia del mejillón, Mytilus galloprovincialis Lamarck 1819, en las costas de la Región del Biobio $\left(36^{\circ} \mathrm{S}\right)$ y de la Región de Los Lagos $\left(54^{\circ} \mathrm{S}\right)$ de Chile (Sanjuán et al. 1997, Tarifeño et al. $2005^{1}$, Toro et al. 2005) suscitó el interés por evaluar la factibilidad biológica, técnica y económica de su cultivo en Chile. Estos aspectos fueron abordados por Proyecto FONDEF D03I-1095 para apoyar el crecimiento de la mitilicultura, incorporando un nuevo recurso cultivable no explotado hasta hoy en el país, conduciendo así a la diversificación de la acuicultura nacional (Tarifeño et al. $2005^{1}$ ). Aun cuando la validez taxonómica de las especies de Mytilus que existen en Chile ha sido materia de recientes estudios genéticos (Toro et al. 2005) y de otros en marcha, la identificación del mejillón sujeto del presente estudio, fue verificado con la aplicación de técnicas estandarizadas de marcadores moleculares para distinguir específicamente entre M. edulis, $M$. galloprovincialis y $M$. trossulus, comprobándose con certeza que se trata de M. galloprovincialis, conocido como mejillón del Mediterráneo (Tarifeño et al. 2005ำ.

${ }^{1}$ Tarifeño E, R Galleguillos, J Gardner, I Lépez, D Arriagada, A Llanos, S Astete, S Ferrada, S Rodríguez \& S Gacitúa. 2005. Presencia del mejillón, Mytilus galloprovincialis (Lmk) (Bivalvia, Mollusca) en las costas de la Región del Biobío, Chile. XXV Congreso de Ciencias del Mar - Colacmar Pontificia Universidad Católica de Valparaíso.
Mytilus galloprovincialis presenta notables ventajas comparativas para su cultivo con respecto a otros mitílidos existentes en las costas chilenas, como son el choro zapato (Choromytilus chorus), la cholgua (Aulacomya atra) y el mejillón chileno (Mytilus edulis chilensis). M. galloprovincialis se destaca sobre estas especies por presentar: i) color amarillo-naranja en ambas gónadas, a diferencia del choro zapato cuya gónada femenina es de color café oscuro (menos atrayente al consumidor); ii) tasa de crecimiento mayor que el mejillón chileno; iii) biso más resistente para el desdoble de cuelgas, y iv) mejores rendimientos en las plantas de proceso (FONDEF D03I-1095). Para desarrollar el cultivo en Chile y así optimizar la producción masiva de semillas en sistemas controlados, se requiere un mejor conocimiento de su ciclo de vida (maduración sexual de reproductores, desarrollo embrionario y desarrollo larval) bajo condiciones ambientales prevalecientes en las costas chilenas. Por ello, se consideró necesario evaluar el efecto de la temperatura en el desarrollo embrionario y larval del mejillón, $M$. galloprovincialis con la finalidad de modular las tasas de crecimiento en sistemas de producción de semillas de este mejillón. El efecto de la temperatura en las tasas de crecimiento larval y tallas de asentamiento ya han sido evaluadas en otros integrantes de la familia Mytilidae, tales como Limnoperna fortuneii kikuchii y Musculista senhousia (Kimura \& Sekiguchi 1996, Cataldo et al. 2005). En ambas especies, las mayores tasas de crecimiento larval se alcanzaron en rangos cálidos.

Considerando que se desconoce la ubicación de bancos naturales de $M$. galloprovincialis que podrían servir como centros de captación masiva de sus semillas, su potencial cultivo en Chile dependerá de la producción de semillas en sistemas controlados, para lo cual es necesario conocer los aspectos biológicos básicos de su reproducción y desarrollo de los estados temprano. Por otra parte, dado que en otras partes del mundo es considerada como una especie invasora en los ecosistemas intermareales (Branch \& Steffani 2004), el estudio de su fase larval contribuiría a entender su dispersión geográfica y desarrollar medidas para el control de su propagación en áreas costeras donde podría entrar en competencia con especies locales. Recientemente, Robinson et al. (2007) indican que la presencia de $M$. galloprovincialis en la costa oeste de Sudáfrica (Branch \& Steffani 2004) puede transformarse en una pesquería sustentable de pequeña escala mediante intensidades de cosecha de $F=0,1$ a 0,3 . 


\section{Material y métodos}

Los reproductores $(>5 \mathrm{~cm})$ de $M$. galloprovincialis se obtuvieron desde el intermareal en la bahía de Coliumo, Chile $\left(36^{\circ} 32^{\prime} \mathrm{S}, 72^{\circ} 57^{\prime} \mathrm{W}\right)$, para su posterior acondicionamiento e inducción a la emisión gamética a través de la técnica de choque térmico (Bayne 1976). Para lograr la liberación de los gametos, los reproductores se depositaron en una bandeja de $10 \mathrm{~L}$ y fueron sometidos a ciclos de 20 minutos de alternancia de agua de mar entre $13^{\circ}$ y $19^{\circ} \mathrm{C}$, previamente filtrada y esterilizada $(1 \mu \mathrm{m}$, UV).

Una vez producida la liberación de los gametos, estos se tamizaron separadamente con malla de $88 \mu \mathrm{m}$, para retener y desechar los restos de materia orgánica. A continuación se les mantuvo durante 20 minutos en agua de mar filtrada para hidratar los ovocitos y activar los espermatozoides. Posteriormente, se realizó la fecundación en una bandeja con $10 \mathrm{~L}$ de agua de mar filtrada a la temperatura estándar aplicada en el criadero de semillas $\left(17^{\circ} \mathrm{C}\right)$. De este cultivo se tomó una muestra de $100 \mathrm{~mL}$ para la caracterización del desarrollo embrionario y el resto se cultivó en condiciones masivas para seguir la observación con el desarrollo larval, el cual se mantuvo hasta la fase de metamorfosis, a $17^{\circ} \mathrm{C}$, aireación suave y constante, dieta en base a la microalga Isochrysis galbana (20.000 células $\mathrm{mL}^{-1}$ primer día y 30.000 células $\mathrm{mL}^{-1}$ a partir del segundo día). Desde el séptimo día se agregó Chaetoceros muelleri en proporción 1:1 con I. galbana. Posteriormente, a partir del día 14 se incorporó a la dieta, Pavlova lutheri (1:1:1), a concentración de 50.000 células $\mathrm{mL}^{-1}$. El recambio de agua de mar filtrada $(1 \mu \mathrm{m})$ y esterilizada con UV se realizó día por medio para eliminar larvas muertas y fecas, con cambio total de agua del cultivo cada 7 días.

El desarrollo embrionario fue seguido mediante la observación de muestras en el microscopio óptico, tomando como referencia las características morfológicas de las fases embrionarias descritas para Choromytilus chorus por Toledo et al. (1990) considerando que la literatura existente para M. galloprovincialis (Satuito et al. 1994) no entrega detalles sobre este proceso. El tiempo de límite entre las fases fue establecido como aquel en el cual el $50 \%$ de la muestra había logrado una fase específica.

Para la caracterización del desarrollo larval se tomaron muestras del cultivo masivo cada dos días, evaluándose en el microscopio óptico, las siguientes condiciones: i) el estado fisiológico general de las larvas mediante la observación de su conducta de natación en la columna de agua (Bayne 1976), ii) presencia de posibles patógenos, iii) acumulación de alimento en el estómago, iv) formación de estructuras larvales. Simultáneamente, se seleccionaron al azar 30 larvas para fijarlas en formalina (5\%) que fueron posteriormente medidas con un ocular graduado (10x). Siguiendo a Ramorino \& Campos (1983) y Rojas (2004) se consideró la longitud valvar (L) como el mayor eje de la concha en sentido paralelo a la charnela y la altura valvar (A) como la mayor distancia entre el extremo del umbo y el borde ventral. La tasa de crecimiento se expresó como un aumento en la longitud de la concha por día (Olavarría et al. 1996).

Para evaluar el efecto de la temperatura sobre el desarrollo embrionario y larval, estos estados fueron mantenidos hasta la metamorfosis en contenedores plásticos de $3 \mathrm{~L}$, los cuales estaban inmersos en baños termorregulados a 12,16 y $20^{\circ} \mathrm{C}$, con un sistema de aireación suave y constante, y dieta basada en mezcla de las microalgas Isochrysis galbana y Chaetoceros muelleri, suministrada a partir del segundo día de inicio del estado larval, y cambio de agua de mar filtrada y esterilizada ( $1 \mu \mathrm{m}, \mathrm{UV})$, día por medio. El procedimiento de control cualitativo y cuantitativo de cada uno de los tratamientos en tres réplicas, fue el mismo indicado anteriormente para el cultivo masivo.

Para verificar si las diferencias observadas en las tasas de crecimiento eran estadísticamente significativas se realizó una determinación de la homocedasticidad de la varianza, seguido por un análisis de covarianza, ANCOVA (Zar 1996). Para verificar en qué temperaturas se producían dichas diferencias se aplicó la prueba $a$ posteriori de Tukey (Zar 1996).

Con el objetivo de comparar el desarrollo temprano de $M$. galloprovincialis con el del mejillón chileno, $M$. edulis chilensis, se realizó una inducción al desove y posterior seguimiento de la fase larval con esta última especie. Se siguió la metodología ya descrita anteriormente con la excepción de la temperatura de incubación que en este caso correspondió a $16^{\circ} \mathrm{C}$.

\section{Resultados}

\section{Desarrollo embrionario y larval de Mytilus galloprovincialis}

El desarrollo embrionario del mejillón, $M$. galloprovincialis comenzó con la formación del cigoto después de la fecundación. La separación de la membrana de fecundación se observó después de 5 minutos, dejando un área transparente entre ella y el contenido del ovocito al separarse de la masa de vitelo (Fig. 1a). Posteriormente, a los 35 minutos, el embrión experimentó las reducciones 


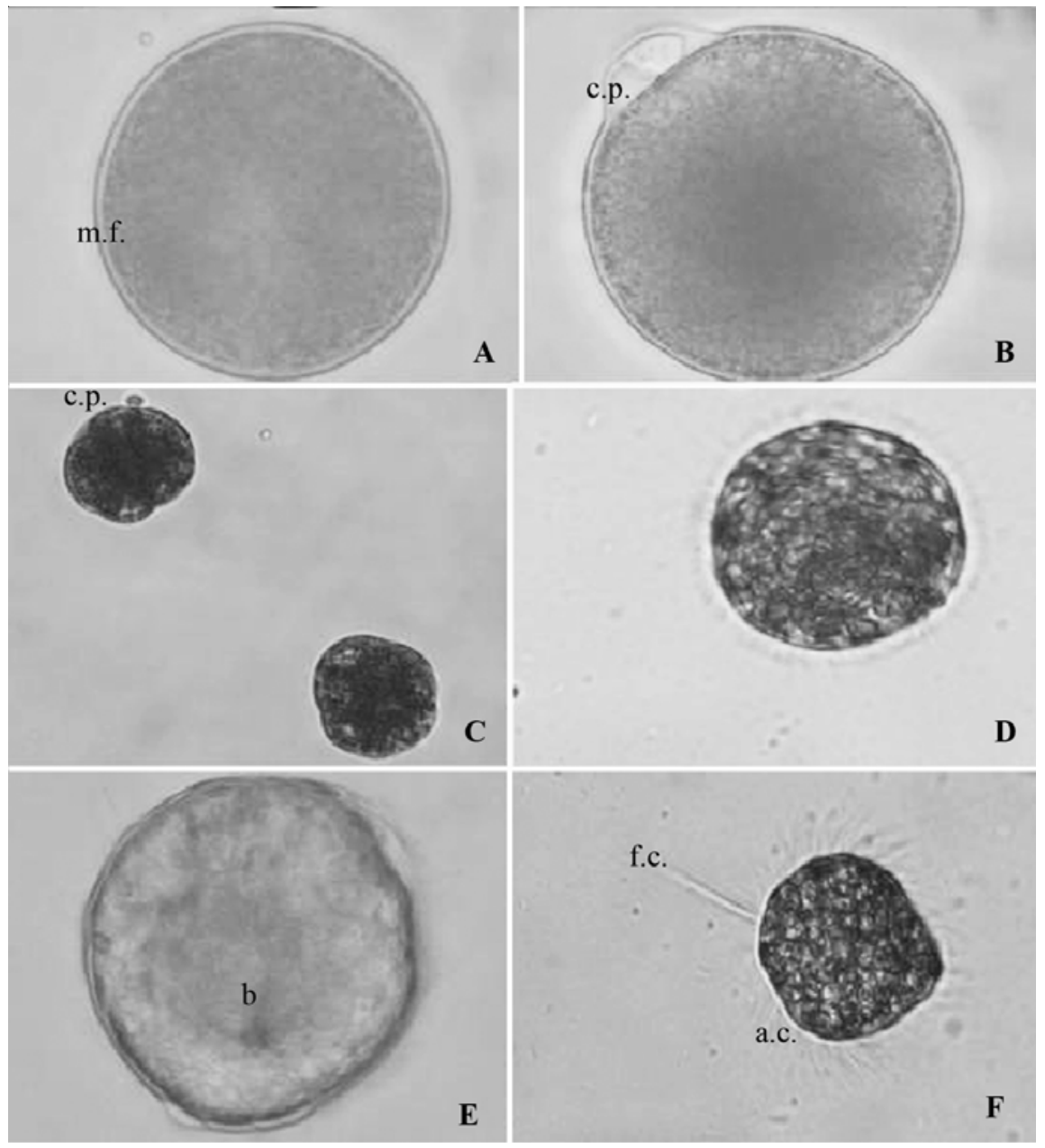

Figura 1

Mytilus galloprovincialis. A. membrana de fecundación (40x), B. aparición de primer corpúsculo polar (40x), C. mórula (10x), D. blástula (10x), E. gástrula (40x) y F. larva trocófora (10x) m.f.: membrana fecundación, c.p.: corpúsculo polar, b: blastoporo, f.c: flagelos centrales, a.c.: anillo ciliado

Mytilus galloprovincialis. A. fertilization membrane (40x), B. first polar corpuscle (40x), C. morula (10x), D. blastula (10x), E. gastrula (40x) and F. trocophore larva (10x) m.f.: fertilization membrane, c.p.: polar corpuscle, b: blastopore, f.c: central flagelates, a.c.: cilial ring

meióticas, con la formación y liberación del primer y segundo corpúsculo polar (Fig. 1b). Después de 40 minutos, sucedió el primer evento mitótico que corresponde a la primera segmentación, con la retención del segundo corpúsculo polar en el eje de segmentación. A continuación, se observó una serie de divisiones celulares que a 4 horas post-fecundación dieron origen al estado de mórula (Fig. 1c), que en dos horas más se convirtió en una blástula, la cual mostró con nitidez el ectodermo y el blastocele. La blástula presentó cilios en toda su superficie que le permitieron nadar en forma rotatoria (blástula rotatoria) (Fig. 1d). A las 7 horas postfecundación, se observó el estado de gástrula, con un blastoporo claramente visible (Fig. 1e). Luego de transcurridas 24 horas, el embrión se convirtió en una larva trocófora, en la cual se distinguió un par de flagelos centrales, de posición anterior o pretrocal, un anillo ciliado en la región media o prototroca y una zona aplanada en la región anterior (Fig. 1f) (Tabla 1).

Larva D: A $17^{\circ} \mathrm{C}$, este estado fue logrado 45 horas postfecundación, con la formación de la primera concha larval o 'prodisoconcha I' con las características valvas en forma D. La longitud y altura máxima valvar se incrementó desde $99 \pm 6 \mu \mathrm{m}$ y $70 \pm 4 \mu \mathrm{m}$, hasta $194 \pm 22 \mu \mathrm{m}$ y 159 $\pm 14 \mu \mathrm{m}$, respectivamente, en el día 10 de edad larval.

Las larvas presentaron un velo ciliado retráctil y un par de flagelos centrales, los que se extendían fuera de 


\section{Tabla 1}

Mytilus galloprovincialis. Tiempos post-fecundación de ocurrencia de las fases del desarrollo embrionario a $17^{\circ} \mathrm{C}$

Mytilus galloprovincialis. Post-fertilization times of the embryonic developmental stages at $17^{\circ} \mathrm{C}$

\begin{tabular}{ll}
\hline \multicolumn{1}{c}{ Estadio } & \multicolumn{1}{c}{ Tiempo post- fecundación } \\
\hline Formación de membrana de fecundación & 5 minutos \\
Liberación del primer corpúsculo polar & 35 minutos \\
Embrión en primera segmentación & 40 minutos \\
Embrión en segunda segmentación & 2 horas \\
Embrión con más de tres divisiones celulares & 2 horas 30 minutos \\
Mórula & 4 horas \\
Blástula & 6 horas \\
Blástula rotatoria & 6 horas 30 minutos \\
Gástrula & 7 horas \\
\hline
\end{tabular}

las valvas durante la natación. En esta fase, el velo ciliado está muy desarrollado y activo. Las larvas mostraron un estómago muy desarrollado ocupando la mayor parte de la cavidad corporal en la región cercana a la charnela, claramente definido y de color café-verdoso por la ingestión de fitoplancton (Fig. 2a).

Larva umbonada: este estado larval fue observado a partir del día 11 post-fecundación y se caracterizó por un crecimiento alométrico de la concha en la región del umbo, provocando de este modo un cambio en la curvatura de la charnela, que dejó de ser una estructura recta, asumiendo un perfil semi-curvo abultado sobre la superficie de las valvas. La longitud y altura máxima promedio valvar al principio de este estado fue de $200 \pm$ $2 \mu \mathrm{m}$ y $168 \pm 2 \mu \mathrm{m}$, respectivamente.
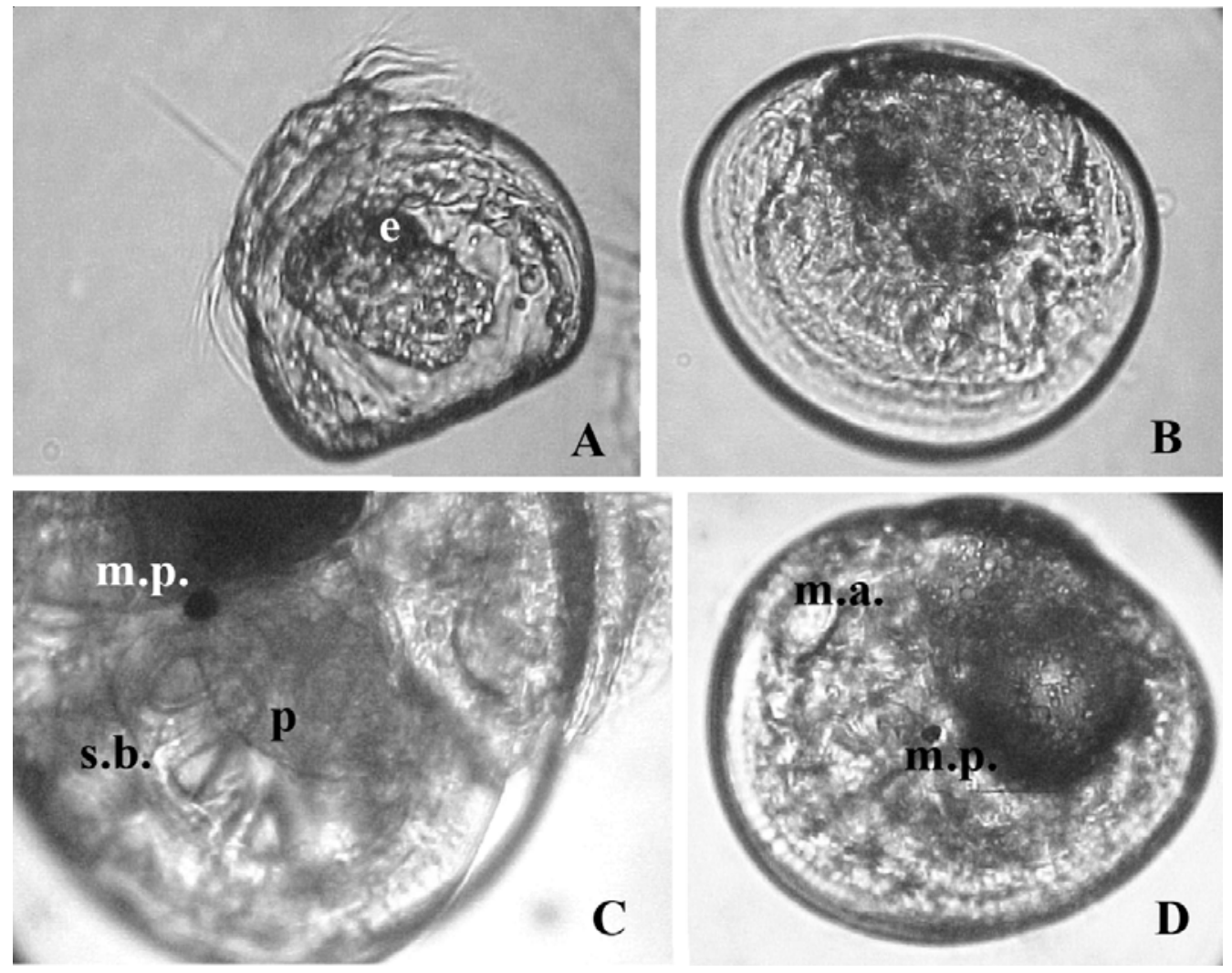

Figura 2

Mytilus galloprovincialis. Desarrollo larval A. velígera en fase D (10x), B. velígera umbonada (10x), C. detalle de una velígera mostrando la mancha pigmentada (10x) y D. pedivelígera (10x) e.: estómago, m.a.: músculos aductores, m.p.: mancha pigmentada, s.b: septos branquiales, v: velo, p: pie

Mytilus galloprovincialis. Larval development A. D-stage veliger (10x), B. umbonate veliger (10x), C. detail of a veliger showing the pigment-spot $(10 \mathrm{x})$ y D. pediveliger (10x): e.: stomach, m.a.: adductor muscle, m.o.: pigment spot, 
Durante esta fase, el tamaño del velo ciliado disminuyó notablemente, al igual que la capacidad natatoria larval en la columna de agua, aunque el movimiento ciliar fue muy activo. El estómago continuó siendo la estructura interna más visible. Al final de esta fase, 15 días post-fecundación, la talla promedio valvar fue de $255 \pm 2 \mu \mathrm{m}$ de longitud y $231 \pm 2 \mu \mathrm{m}$ de altura. Se observaron claramente además, las inserciones de los músculos aductores y una mancha pigmentada oscura conocida como la mancha ocular, en la parte central de los tejidos blandos en inmediatamente debajo de la valva (Fig. 2b).

Larva pedivelígera: esta fase se inició a los 17 días postfecundación. Las larvas presentaron una longitud y altura máxima promedio valvar de $265 \pm 2 \mu \mathrm{m}$ y $247 \pm 1 \mu \mathrm{m}$, respectivamente. Esta fase larval se caracterizó por la presencia de un pié bien desarrollado y funcional, que era continuamente extendido fuera de las valvas. También se inició la formación de varios septos branquiales y se observó una notable disminución del tamaño del velo (Fig. 2c y 2d). Este estado se completó el día 21 postfecundación, con un comportamiento larval de muy poca actividad natatoria, preferentemente en el fondo de los estanques, pero con gran actividad del pié digitiforme; la longitud y altura máxima promedio valvar fueron 281 $\pm 3 \mu \mathrm{m}$ y $289 \pm 3 \mu \mathrm{m}$, respectivamente.
En la Tabla 2 se muestra una comparación que resume las principales características de cada fase larval. Con el estadio de pedivelígera termina el desarrollo larval, entrando la larva a una condición premetamórfica.

La curva de crecimiento larval en el cultivo masivo a $17^{\circ} \mathrm{C}$, se ajustó a una regresión lineal (longitud valvar $=$ $10,6 \mu \mathrm{m}^{*} \mathrm{~d}^{-1} *$ edad $\left.+75,4\right)$; sin embargo, presentó dos fases que se separaron en el día 15 del cultivo que corresponde al inicio de la metamorfosis. Por este motivo se determinaron dos tasas de crecimiento, una por cada fase de la curva, presentando la primera una pendiente de $12,1 \mu \mathrm{m}^{*}$ día $^{-1}$ y la segunda, incrementos de 5,5 $\mu \mathrm{m}^{*}$ día $^{-1}$ (Fig. 3).

\section{Efecto de la temperatura sobre el desarrollo} embrionario y larval de Mytilus galloprovincialis

El desarrollo embrionario de $M$. galloprovincialis a los 12,16 y $20^{\circ} \mathrm{C}$, mostró diferencias significativas en los tiempos de desarrollo. En general, todos los procesos embrionarios fueron más rápidos a $20^{\circ} \mathrm{C}$, seguido por los $16^{\circ} \mathrm{C}$, y finalmente a $12^{\circ} \mathrm{C}$ (Tabla 3 ). A $20^{\circ} \mathrm{C}$, a las $18,5 \mathrm{~h}$ post-fecundación se observó la primera fase larval de trocófora, mientras que a 12 y $16^{\circ} \mathrm{C}$, esta fase larval se alcanzó a las 27 y 23,5 h, respectivamente.

Tabla 2

Mytilus galloprovincialis. Características morfológicas y de comportamiento de los estadios larvales Mytilus galloprovincialis. Morphological and behavior characteristics of the larval stages

\begin{tabular}{|c|c|c|}
\hline Larva D & Larva umbonada & Larva pedivelígera \\
\hline Prodisoconcha I y II & Prodisoconcha I y II & Prodisoconcha I y II \\
\hline Charnela recta & Charnela semi-curva & Charnela semi-curva \\
\hline Estrías concéntricas & Estrías concéntricas & Estrías concéntricas \\
\hline Estómago bien definido & Estómago bien definido & $\begin{array}{l}\text { Estómago definido, excepto al } \\
\text { final del estadio }\end{array}$ \\
\hline Velo ciliado funcional & Velo en regresión & Velo en regresión avanzada \\
\hline \multirow[t]{4}{*}{ Presencia de un par de flagelos } & Ausencia de flagelos & Ausencia de flagelos \\
\hline & $\begin{array}{l}\text { Inserciones de músculos aductores } \\
\text { visibles }\end{array}$ & $\begin{array}{l}\text { Inserciones de músculos aductores } \\
\text { bien definidas }\end{array}$ \\
\hline & Septos branquiales en desarrollo & $\begin{array}{l}\text { Septos branquiales bien } \\
\text { desarrollados }\end{array}$ \\
\hline & Presencia de mancha pigmentada & $\begin{array}{l}\text { Presencia de mancha pigmentada. } \\
\text { Órgano pedal funcional }\end{array}$ \\
\hline $\begin{array}{l}\text { Larva nadadora muy activa en la } \\
\text { columna de agua }\end{array}$ & $\begin{array}{l}\text { Disminución de la capacidad } \\
\text { natatoria en la columna de agua }\end{array}$ & $\begin{array}{l}\text { Larva con poca o nula actividad } \\
\text { natatoria, preferentemente sésil }\end{array}$ \\
\hline
\end{tabular}


Tabla 3

Mytilus galloprovincialis. Tiempo de desarrollo de las fases embrionarias a 12,16 y $20^{\circ} \mathrm{C}$

Mytilus galloprovincialis. Development time of embryonic stages at 12,16 and $20^{\circ} \mathrm{C}$

Proceso embrionario

Temperatura

\begin{tabular}{llll} 
& \multicolumn{1}{c}{$12^{\circ} \mathrm{C}$} & \multicolumn{1}{c}{$16^{\circ} \mathrm{C}$} & \multicolumn{1}{c}{$20^{\circ} \mathrm{C}$} \\
\hline Membrana de fecundación & 10 minutos & 5 minutos & 5 minutos \\
Primer corpúsculo polar & 60 minutos & 40 minutos & 15 minutos \\
Primera segmentación & 2 horas & 1 hora 30 minutos & 1 hora 20 minutos \\
Segunda segmentación & 3 horas 20 minutos & 2 horas 20 minutos & 1 hora 40 minutos \\
Embriones $>3$ & 4 horas & 3 horas & 2 horas \\
segmentaciones & 5 horas 30 minutos & 3 horas 30 minutos & 2 horas 20 minutos \\
Mórula & 7 horas & 5 horas 30 minutos & 4 horas \\
Blástula & 8 horas & 7 horas & 5 horas 30 minutos \\
Blástula rotatoria & & 23 horas 30 minutos & 18 horas 30 minutos \\
Fin de la fase embrionaria & 27 horas & & \\
aparición de trocófora & &
\end{tabular}

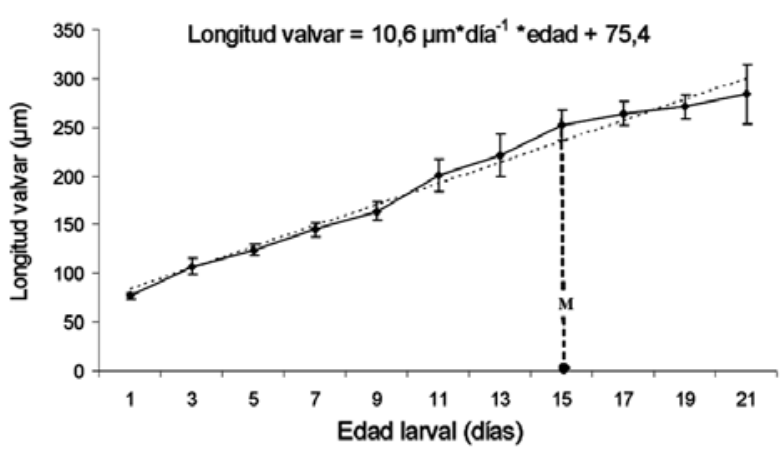

Figura 3

Mytilus galloprovincialis. Crecimiento larval en cultivo masivo en criadero a $17^{\circ} \mathrm{C}$. M: metamorfosis

Mytilus galloprovincialis. Larval growth in hatchery mass culture at $17^{\circ} \mathrm{C} . \mathrm{M}$ : metamorphosis

Las larvas cultivadas a 12,16 y $20^{\circ} \mathrm{C}$ presentaron una tasa de crecimiento muy similar durante los primeros 8 días, con medidas morfométricas muy próximas entre sí. Sin embargo, el efecto de la temperatura fue evidente a partir del día 8 (Tabla 4). Así, las larvas, a $20^{\circ} \mathrm{C}$, alcanzaron los estados de larva $\mathrm{D}$, larva umbonada y pedivelígera en un menor tiempo de desarrollo que las larvas cultivadas a 12 y $16^{\circ} \mathrm{C}$. Las tasas de crecimiento en longitud valvar entre las temperaturas de cultivo ensayadas, fueron estadísticamente distintas (ANCOVA
$\mathrm{F}=561,89, P>0,001$ ), con valores de $6,7 \mu \mathrm{m}^{*} \mathrm{dí}^{-1}$ a $12^{\circ} \mathrm{C}$, de $7,4 \mu \mathrm{m}^{*}$ día $^{-1}$ a $16^{\circ} \mathrm{C}$ y de $9,1 \mu \mathrm{m}^{*}$ día $^{-1}$ a $20^{\circ} \mathrm{C}$ (Fig. 4). La prueba a posteriori indicó una diferencia a los $20^{\circ} \mathrm{C}$ con respecto de las temperaturas de 12 y $16^{\circ} \mathrm{C}$ (Tuckey $\mathrm{F}=561,89 ; P<0,01)$; sin embargo, estas últimas no presentaron diferencias significativas entre sí (Tuckey $\mathrm{F}=561,89 ; P>0,01)$. Similares resultados se evidenciaron al considerar la tasa de crecimiento para el eje valvar de altura.

\section{Desarrollo larval de Mytilus edulis chilensis}

La morfología larval de M. edulis chilensis no mostró diferencias con respecto a lo descrito anteriormente para las larvas de $M$. galloprovincialis. Las larvas D de $M$. edulis chilensis, con longitud inicial de $108 \pm 5 \mu \mathrm{m}$ y alto de $76 \pm 8 \mu \mathrm{m}$, finalizaron esta fase el día 11 post fecundación, con longitudes de $166 \pm 7 \mu \mathrm{m}$ y alturas de $129 \pm 6 \mu \mathrm{m}$. La aparición de la fase umbonada se inició el día 9, predominando este estado a partir del día 13. Al inicio de esta etapa las larvas midieron $169 \pm 7 \mu \mathrm{m}$ de largo y $134 \pm 13 \mu \mathrm{m}$ de alto, finalizando el desarrollo larval día 19 post fecundación, con una talla promedio de $190 \pm 13 \mu \mathrm{m}$ de longitud y de $157 \pm 12 \mu \mathrm{m}$ de altura.

$\mathrm{El}$ crecimiento larval de M. edulis chilensis a $16^{\circ} \mathrm{C}$ presentó una tendencia similar a lo observado para $M$. galloprovincialis (Fig. 5). La tasa de crecimiento larval, previo al proceso de metamorfosis (día 15), alcanzó igual magnitud en ambas especies (M. edulis chilensis: $\mathrm{y}=5,3 \mathrm{x}$ 
Tabla 4

Mytilus galloprovincialis. Longitud total promedio ( \pm d.e.) de larvas cultivadas a 12,16 y $20^{\circ} \mathrm{C}(\mathrm{n}=30)$

Mytilus galloprovincialis. Mean total length ( \pm s.d.) of larvae reared at 12,16 y $20^{\circ} \mathrm{C}(\mathrm{n}=30)$

\begin{tabular}{cccc}
\hline \multirow{2}{*}{ Edad larval (días) } & $12^{\circ} \mathrm{C}$ & $\begin{array}{c}\text { Longitud valvar }(\mu \mathrm{m}) \\
16^{\circ} \mathrm{C}\end{array}$ & $20^{\circ} \mathrm{C}$ \\
\hline 2 & $110 \pm 5$ & $110 \pm 5$ & $110 \pm 5$ \\
4 & $116 \pm 6$ & $123 \pm 3$ & $123 \pm 4$ \\
6 & $119 \pm 6$ & $132 \pm 5$ & $132 \pm 2$ \\
8 & $129 \pm 6$ & $134 \pm 4$ & $158 \pm 5$ \\
10 & $136 \pm 11$ & $142 \pm 6$ & $167 \pm 3$ \\
12 & $146 \pm 12$ & $166 \pm 7$ & $180 \pm 7$ \\
14 & $164 \pm 9$ & $171 \pm 7$ & $191 \pm 11$ \\
16 & $183 \pm 9$ & $186 \pm 7$ & $229 \pm 11$ \\
18 & $192 \pm 12$ & $214 \pm 7$ & \\
20 & $210 \pm 14$ & $239 \pm 9$ & $\mathbf{9}$ (fijación larval) \\
22 & $225 \pm 11$ & $\mathbf{2 6 3} \pm \mathbf{1 3}$ (fijación larval) & \\
24 & $\mathbf{2 5 1} \pm \mathbf{1 5}$ (fijación larval) & & \\
\hline
\end{tabular}

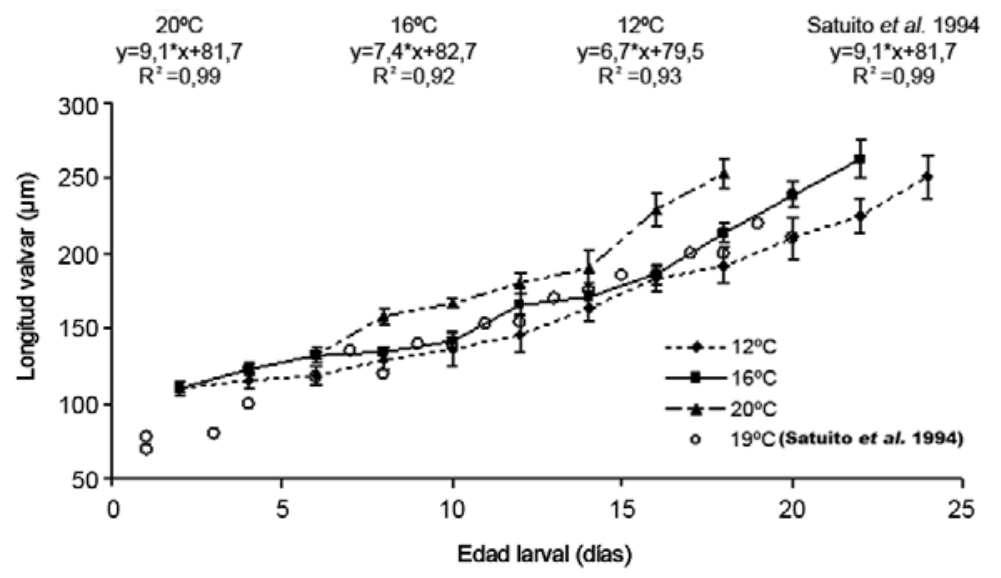

Figura 4

Mytilus galloprovincialis. Crecimiento larval a 12, 16 y $20^{\circ} \mathrm{C}$

Mytilus galloprovincialis. Larval growth at 12, 16 and $20^{\circ} \mathrm{C}$

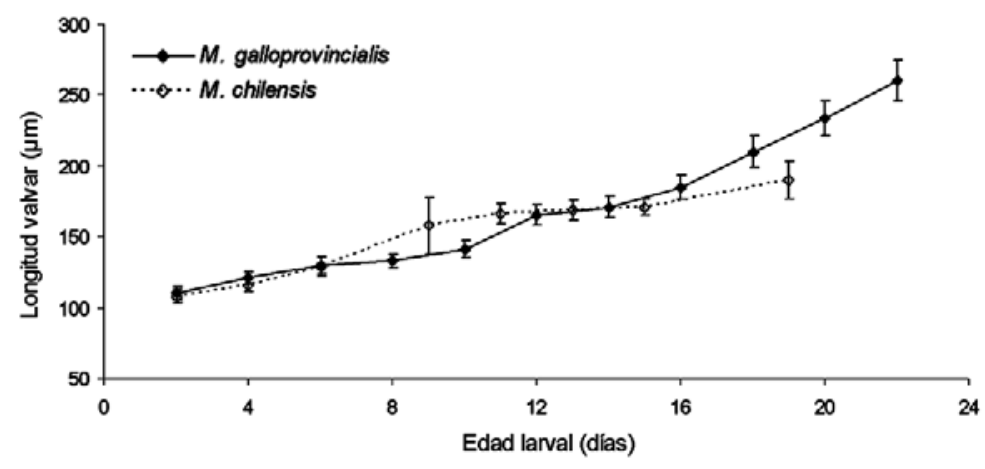

Figura 5

Crecimiento larval de Mytilus galloprovincialis y Mytilus edulis chilensis a $16^{\circ} \mathrm{C}$

Mytilus galloprovincialis and Mytilus edulis chilensis larval growth at $16^{\circ} \mathrm{C}$ 
+ 98,9, $\mathrm{R}^{2}=0,89$ y M. galloprovincialis: $\mathrm{y}=5,2 \mathrm{x}+98,3$ $\left.\mathrm{R}^{2}=0,91\right)$. A partir de la metamorfosis se observó una menor tasa de crecimiento en comparación con $M$. galloprovincialis.

\section{Discusión}

El desarrollo ontogénico del mejillón, Mytilus galloprovincialis con una duración total aproximada de 21 días a $17^{\circ} \mathrm{C}$, generó embriones y larvas que presentan las características típicas descritas para los mejillones de la familia Mytilidae (Bayne 1976, Le Pennec 1978, Ramorino \& Campos 1983, Chaparro \& Sanhueza 1986, Toledo et al. 1990). Este antecedente de tiempo al cual ocurre la metamorfosis indica una ventaja comparativa para M. galloprovincialis, desde el punto de vista de su cultivo respecto a otros mitílidos en las costas de Chile, como el choro zapato, Choromytilus chorus, en el cual esta misma fase de desarrollo larval se alcanza en 25 días a $16,5 \pm 0,5^{\circ} \mathrm{C}$ (Chaparro \& Sanhueza 1986). El menor tiempo para lograr la metamorfosis corresponde con la alta tasa de crecimiento mostrada por $M$. galloprovincialis, que registró incrementos de 12,1 $\mu \mathrm{m}^{*}$ día $^{-1}$ para los primeros 15 días y de $5,5 \mu \mathrm{m}^{*}$ día $^{-1}$ para los subsiguientes días de desarrollo, lo que indica una marcada desaceleración del crecimiento durante la fase final del desarrollo larval. Whyte et al. (1990) señalan que en el ostión, Crassadoma gigantea ocurre una baja en la eficiencia del crecimiento al acercarse la fase de metamorfosis como consecuencia de la necesidad larval de almacenar los lípidos para la metamorfosis y por lo tanto los resta del gasto energético para el crecimiento larval. En el caso de M. galloprovincialis, parece ocurrir lo mismo dado que las tasas de crecimiento disminuyeron notablemente al acercarse la fase de metamorfosis.

Ramorino \& Campos (1983), utilizando como criterio de clasificación la talla a la cual ocurre la metamorfosis (mayor o menor a $240 \mu \mathrm{m}$ ), separan en dos grupos a los seis representantes de la familia Mytilidae presentes en Chile. Las larvas de Semimytilus algosus, Perumytilus purpuratus y Brachidontes granulata entrarían en la fase de metamorfosis a tallas $<230 \mu \mathrm{m}$, mientras que Mytilus edulis chilensis, Choromytilus chorus y Aulacomya atra lo harían a tallas $>240 \mu \mathrm{m}$. La talla de metamorfosis de $265 \mu \mathrm{m}$ observada en M. galloprovincialis lo ubicaría dentro del segundo grupo de mitílidos coincidiendo con lo indicado por Ramorino \& Campos (1983) respecto a la talla mínima para la metamorfosis.

Ockelmann (1965) relaciona el tamaño del ovocito, de la prodisoconcha I (P I) y de la prodisoconcha II (P II) con el tipo de desarrollo de una especie, lo cual es de gran valor al momento de identificar los primeros estados

\section{Tabla 5}

Diámetro de ovocito, longitud de prodisoconcha I (P I) y prodisoconcha II (P II) de especies de Mytilidae de la costa chilena

Oocyte diameter, prodissoconch I (P I) and prodissoconch II (P II) length of Mytilidae species from the Chilean coast

\begin{tabular}{lccc}
\hline & Ovocito $(\mu \mathrm{m})$ & P I $(\mu \mathrm{m})$ & P II $(\mu \mathrm{m})$ \\
\hline Mytilus galloprovincialis & $65,0-75,0$ & $75,0-107,5$ & $162,0-217,5$ \\
Choromytilus chorus & $63,7-68,0$ & $93,5-108,4$ & $304,5-362,0$ \\
Aulacomya atra & $70,1-78,6$ & $105,0-121,1$ & $315,0-420,0$ \\
Mytilus edulis chilensis & $64,8-70,1$ & $102,0-116,9$ & $262,5-341,2$ \\
Semimytilus algosus & $68,3-78,7$ & $119,0-136,0$ & $183,8-216,8$ \\
Brachidontes gramulata & $63,0-73,0$ & $114,8-132,0$ & $193,4-231,0$ \\
Perumytilus purpuratus & $115,0-131,2$ & $182,0-197,6$ & $196,2-199,5$ \\
\hline
\end{tabular}

del desarrollo entre especies de una misma familia. $M$. galloprovincialis presenta una pequeña talla de P I, propio de especies con desarrollo planctotrófico; además, presenta el rango de longitud de P I más pequeño comparado con otras especies de la familia Mytilidae de la costa chilena (Tabla 5). Esta característica, junto con la morfología de la concha permite su identificación específica, lo que es de importancia para estudios de dinámica larval y postlarval en áreas naturales donde $M$. galloprovincialis puede coexistir con alguna de las otras especies de mitílidos de la costa de Chile. La inclusión de M. galloprovincialis dentro de los patrones referidos por Ramorino \& Campos (1983), es avalada además, por la gran similitud existente entre el desarrollo larval de estas especies en cuanto a: i) tipo de estructuras presentes, tales como velo desarrollado, flagelo central, una mancha ocular muy notoria, ii) la forma y estructura de la charnela, la cual es recta en su inicio y semi-curva al final del estado de pedivelígera, y iii) las tallas alcanzadas durante cada fase (Cáceres 1981, Chaparro \& Sanhueza 1986).

Los estudios referentes al efecto de la temperatura sobre el desarrollo embrionario o larval en bivalvos señalan que estos procesos no sufren alteraciones morfológicas dentro de estrechos rangos de temperatura, respecto a las subsiguientes etapas del desarrollo ontogénico (Loosanoff \& Davis 1963, Calabrese \& Davis 1970). Sin embargo, en los experimentos de temperatura realizados en este estudio se observó que los embriones y larvas de $M$. galloprovincialis sometidas al rango entre 12 y $20^{\circ} \mathrm{C}$ fueron capaces de sobrevivir y continuar su desarrollo sin alteraciones morfológicas visibles. El efecto de la temperatura se evidenció sólo en la duración de las fases embrionaria y larval. Hubo una notable disminución del tiempo de duración de los estados embrionarios: $46 \%$ a $20^{\circ} \mathrm{C}$ y $27 \%$ a $16^{\circ} \mathrm{C}$, respecto a lo 
estimado a $12^{\circ} \mathrm{C}$. Esta misma relación sinérgica de temperatura sobre la velocidad de desarrollo fue descrita por Hrs-Brenko (1975) para embriones de M. edulis de la costa este de EUA y para M. galloprovincialis del Mar Adriático, cuyo desarrollo embrionario normal ocurrió en un rango de $15-20^{\circ} \mathrm{C}$, coincidente con los observado en el presente estudio para esta especie. De igual modo, los resultado del presente estudio son similares a los registrados por His et al. (1989) para M. galloprovincialis proveniente de la bahía de Arcachon (Francia) y por Beaumont et al. (2004) que mostraron que larvas velígeras de este mejillón crecieron a mayor velocidad a $20^{\circ} \mathrm{C}$, en relación a 14 y $10^{\circ} \mathrm{C}$. Sin embargo, las larvas de M. galloprovincialis sometidas a $16^{\circ} \mathrm{C}$ en el presente estudio presentaron un crecimiento superior $(7,4$ $\mu \mathrm{m}^{*}$ día $\left.^{-1}\right)$ a la tasa estimada por Pechenik et al. (1990) para $M$. edulis $\left(3,1 \mu \mathrm{m}^{*}\right.$ día $\left.^{-1}\right)$ a igual temperatura.

Considerando que los datos disponibles para $M$. galloprovincialis sobre crecimiento a distintas temperatura (His et al. 1989, Beaumont et al. 2004) no tuvieron como objetivo establecer tasas de crecimiento larval, no es posible hacer comparaciones específicas respecto al efecto de la temperatura en distintas poblaciones de esta especie. Satuito et al. (1994) entregan información sobre crecimiento larval a $19 \pm 1^{\circ} \mathrm{C}$, la cual comparada con nuestros resultados es sólo similar a la tasa obtenida a $16^{\circ} \mathrm{C}$ (Fig. 4). Esta mayor tasa de crecimiento larval en temperaturas más bajas sugiere una adecuada ventaja para el cultivo de $M$. galloprovincialis en Chile, considerando que las aguas de la zona costera centro-sur están en un rango térmico de $12-15^{\circ} \mathrm{C}$.

Tradicionalmente se menciona que $M$. galloprovincialis es una especie de aguas temperadas cálidas; sin embargo, en Chile no existen, hasta la fecha, referencias de la presencia de $M$. galloprovincialis al norte de la bahía de Coliumo $\left(36^{\circ} \mathrm{S}\right)$, aunque se han realizado prospecciones preliminares hasta las costas de Iquique $\left(20^{\circ} \mathrm{S}\right) . \mathrm{Su}$ presencia ha sido ratificada genéticamente solo desde el Estrecho de Magallanes hasta el Golfo de Arauco (Toro et al. 2005, Cárcamo et al. 2005, Tarifeño et al. 2005). Actualmente, se conoce que este mejillón tiene más bien una distribución mundial cosmopolita (Gardner 2004, Sanjuán et al. 1997) y por lo tanto su rango de tolerancia térmica podría tener una distribución más amplia. Tomando en cuenta que los resultados experimentales mostraron un desarrollo embrionario y larval exitoso a temperaturas de 12 a $20^{\circ} \mathrm{C}$, su ausencia en las costas chilenas al norte de los $36^{\circ} \mathrm{S}$ podría ser determinada por factores distintos al efecto de la temperatura.

Si bien es cierto que la existencia en Chile de $M$. galloprovincialis había sido indicada ya en 1997 (Sanjuán et al. 1997), la certificación genética de su presencia fue confirmada recién en 2005 (Toro et al. 2005, Tarifeño et al. $2005^{1}$ ). Su presencia en las costas chilenas podrían corresponder a un patrón de dispersión de esta especie en ecosistemas costeros donde estaría actuando como una especie invasora, como ha sido descrita para la costa oeste de Sudáfrica (Branch \& Steffani 2004). Sin embargo, Robinson et al. (2007) indican que la abundancia de esta especie en dichas costas puede transformarse en una gran oportunidad para desarrollar una pesquería de pequeña escala sustentable con intensidades de cosecha de $F=$ 0,1 a 0,3 con impacto económico y social positivo en las empobrecidas comunidades de pescadores artesanales. Por lo anterior, se podría fomentar el cultivo de esta especie en áreas costeras donde no existen poblaciones naturales de $M$. chilensis y/o Ch. chorus, con las cuales eventualmente podría entrar en competencia espacial.

\section{Agradecimientos}

El financiamiento para la ejecución del presente estudio fue provisto por el Proyecto FONDEF D03I-1095, desarrollado por el Grupo ProMytilus de la Universidad de Concepción. Se agradece al personal técnico y profesional del proyecto su eficiente colaboración en las labores rutinarias del cultivo masivo de larvas de $M$. galloprovincialis en la Unidad Productora de Semillas (UPROS) en la Estación de Biología Marina de la Universidad de Concepción, en Dichato (Región del Biobío, Chile) y a L. González, técnico del Laboratorio de Malacología de la Universidad de Valparaíso, por su ayuda en el análisis morfométrico de postlarvas.

\section{Literatura citada}

Bayne B. 1976. Marine mussels, their ecology and physiology, 506 pp. Cambridge University Press, London.

Beaumont A, G Turner, A Word \& D Skibinski. 2004. Hybridations between Mytilus edulis and Mytilus galloprovincialis and performance of pure species and hybrid veliger larvae at different temperatures. Journal of Experimental Marine Biology and Ecology 302: 177-188.

Blanco J, M Bermúdez, F Arévalo, C Salgado \& Á Moroño. 2002. Depuration of mussels (Mytilus galloprovincialis) contaminated with domoic acid. Aquatic Living Resources 15: $53-60$.

Branch G \& C Steffani. 2004. Can we predict the effects of alien species? A case-history of the invasion of South Africa by Mytilus galloprovincialis (Lamarck). Journal of Experimental Marine Biology and Ecology 300: 189-215.

Cáceres O. 1981. Presencia y distribución de larvas de Mytilus chilensis Hupe 1854 en estero Yaldad ( $43^{\circ} 08^{\prime} \mathrm{S}$; $\left.73^{\circ} 43^{\prime} \mathrm{W}\right)$ y algunas características hidrográficas durante el período estival 1981, Chiloé Chile. Tesis de Biología Marina. Universidad de Concepción, Concepción, 53 pp. 
Calabrese A \& H Davis. 1970. Tolerances and requirements of embryos and larvae of bivalve molluscs. Helgolander Wissenschftliche Meeresuntersuchungen 20: 553-564.

Cárcamo C, Á Comesaña, F Winkler \& A Sanjuán. 2005. Allozyme identification of mussels (Bivalvia: Mytilus) on the Pacific coast of South America. Journal of Shellfish Research 24(4): 1101-1115.

Cataldo D, D Boltovskoy, J Hermosa \& C Canzi. 2005. Temperature-dependent rates of larval development in Limnoperna fortunei (Bivalvia: Mytilidae). Journal of Molluscan Studies 71(1): 41-46.

Chaparro O \& M Sanhueza. 1986. Desarrollo embrionario y larval del choro zapato Choromytilus chorus (Bivalvia, Mytilidae). Biología Pesquera 15: 75-79.

Gardner J. 2004. A historical perspective of the genus Mytilus (Bivalvia: Mollusca) in New Zealand: multivariate morphometric analyses of fossil, midden and contemporary blue mussels. Biological Journal of the Linnean Society 82(3): 329-334.

His E, R Robert \& A Dinet. 1989. Combined effects of temperature and salinity on fed and starved larvae of the Mediterranean mussel Mytilus galloprovincialis and the Japanese oyster Crassostrea gigas. Marine Biology 100: 455-463.

Hrs-Brenko M. 1975. The study of mussel larvae and their settlement in Vela Draga Bay (Pula, The Northern Adriatic Sea). Aquaculture 2: 179-82.

Kimura T \& H Sekiguchi. 1996. Effect of temperature on larval development of two mytilid species and their implication. Venus 55(3): 215-222.

Le Pennec M. 1978. Genèse de la coquille larvaire et post larvaire chez divers bivalves marins. Thèse d'Etat, Université de Bretagne Occidentale, Brest, 229 pp.

Loosanoff V L \& H C Davies. 1963. Rearing of bivalve molluscs. Advances in Marine Biology 1: 1-136.

Ockelmann K. 1965. Developmental types in marine bivalves and their distribution along the Atlantic coast of Europe. Proceedings of the First European Malacological Congress (1962): 25-35.

Olavarria E, A Farías \& I Uriarte. 1996. Morfometría y tasas de crecimiento larvario y post-larvario de los bivalvos Venus antiqua (King \& Broderip, 1835) y Gari solida (Gray, 1828) cultivadas en laboratorio. Revista de Biología Marina 31(2): 107-116.

Pechenik J, L Eyster, J Widdows \& B Bayne. 1990. The influence of concentration and temperature on growth and morphological differentiation of blue mussel Mytilus edulis L. larvae. Journal of Experimental Marine Biology and Ecology 136: 47-64.

Ramorino L \& B Campos. 1979. Desarrollo larval y postlarval de Perumytilus purpuratus (Lamarck, 1819), Bivalvia: Mytilidae. Anales del Museo de Historia Natural, Valparaíso 12: 207-218.

Ramorino L \& B Campos. 1983. Larvas y post-larvas de Mytilidae de Chile (Mollusca: Bivalvia). Revista de Biología Marina 19(2): 143-193.

Robinson T, A Govender, Ch Griffiths \& G Branco. 2007. Experimental harvesting of Mytilus galloprovincialis: Can an alien mussel support a small-scale fishery? Fisheries Research 88: 33-41.

Rojas H. 2004. Desarrollo larval y crecimiento de juveniles post-metamórficos de Tagelus dombeii (Lamarck, 1818) (Bivalvia: Tellinacea: Solecurtidae) en sistema controlado. Tesis de Biología Marina. Universidad Católica del Norte. Coquimbo, $52 \mathrm{pp}$.

Sanjuán A, C Zapata \& G Álvarez. 1997. Genetic differentiation in Mytilus galloprovincialis Lmk. throughout the world. Ophelia 47(1): 13-31.

Satuito G, K Natoyama, M Yamazaki \& N Fusetani. 1994. Larval development of the mussel Mytilus galloprovincialis cultured under laboratory conditions. Fisheries Science 60(1): 65-68.

SERNAPESCA. 2005. Anuario estadístico de Pesca 2004. 187 pp. Servicio Nacional de Pesca, Valparaíso.

SUBPESCA. 2005. Informe Consolidado de Pesca y Acuicultura. Departamento de Análisis Sectorial. 20 pp., Subpesca, Valparaíso.

Toledo P, G Bellolio \& E Dupré. 1990. Fecundación y desarrollo embrionario de Choromytilus chorus (Molina, 1782) (Bivalvia, Mytilidae). Revista de Biología Marina 25(2): 47-57.

Toro J, J Ojeda, AM Vergara, G Castro \& AAlcalán. 2005. Molecular characterization of the Chilean blue mussel (Mytilus chilensis Hupe 1854) demonstrates evidence for the occurrence of Mytilus galloprovincialis in southern Chile. Journal of Shellfish Research 24 (4): 1117-1122.

Whyte J, N Bourne \& NG Ginther. 1990. Biochemical and energy changes during embryogenesis in the rock scallop Crassadoma gigantea. Marine Biology 106: 239-244.

Zar JH. 1996. Biostatistical analysis, 276 pp. Prentice-Hall, New Jersey.

Recibido el 9 de marzo de 2007 y aceptado el 30 de noviembre de 2007 